(To appear in The Astronomical Journal, September 1998)

\title{
An Optical Multicolor System For Measuring Galaxy Redshifts And Spectral Types
}

\author{
Charles T. Liu ${ }^{1}$ and Richard F. Green \\ National Optical Astronomy Observatories, Box 26732, Tucson, AZ 85726
}

\begin{abstract}
A method of obtaining approximate redshifts and spectral types of galaxies using a photometric system of six broad-bandpass filters is developed. The technique utilizes a smallest maximum difference approach rather than a least-squares approach, and does not consider a galaxy's apparent magnitude in the determination of its redshift. In an evalution of its accuracy using two distinct galaxy samples, the photometric redshifts are found to have a root mean square deviation of \pm 0.05 from spectroscopically determined redshifts. Possible systematic errors of the method are investigated, including the effects of post-starburst ("E+A") galaxies and attempts to measure redshifts with incomplete color information. Applications of the technique are discussed.
\end{abstract}

Subject headings:

\section{Introduction}

The traditional method of obtaining redshifts through spectroscopy, though accurate, is by far the most time-consuming task in any observational study of faint extragalactic objects. This is especially true for galaxies, most of which do not have spectral features so dominant as to lend themselves to quick, unambiguous redshift measurements. The need to obtain large numbers of redshifts for large samples of galaxies, however, has never been greater. Many have therefore attempted to design and refine methods to obtain accurate redshifts of galaxies with very low spectral resolution (see, e.g., Koo 1985; Loh \& Spillar 1986; Connolly et al. 1995; Sawicki, Lin \& Yee 1997; Brunner et al. 1997).

We present here a photometric redshift technique we have developed, using template galaxy spectral energy distributions (SEDs) and a system of six optical and near-infrared broad-band

\footnotetext{
${ }^{1}$ Current address: Department of Astronomy, Columbia University, 550 W 120 St, New York, NY 10027
} 
filters. The technique relies on a smallest maximum difference approach rather than a least-squares method, and is optimized for the analysis of galaxy data obtained from the deep multicolor survey of Hall et al. (1996a, hereafter HOGPW). The results of this work are being applied to the data in a companion paper (Liu et al. 1998).

\section{Model Galaxies and Colors}

The six-band filter system of HOGPW consists of standard Johnson U, B and V filters, and non-standard R and I filters (called R, I75, and I86) with approximate effective wavelengths of $6615 \AA, 7425 \AA$, and $8586 \AA$ respectively, and FWHM of $\sim 1000 \AA$ each. Together, the system covers the wavelength range $3000-9000 \AA$ in approximately $1000 \AA$ intervals. The detector response included in the model colors was also that of the one used in the HOGPW survey, i.e. a Textronix $2048 \times 2048$ CCD. The transmission functions of the filters, and the quantum efficiency curve of the CCD, are presented in Figure 1.

Five representative galaxy templates are used in this photometric redshift scheme. Four of the models are taken from the integrated spectrophotometry of Kennicutt (1992) and Coleman, Wu \& Weedman (1980), for spectra representative of E/S0, Sbc, Scd and Irr (starburst) galaxies. The higher-resolution spectra from Kennicutt (1992) were spliced together with the Coleman et al. data - NGC 5248 with the Sbc galaxy, NGC 6181 with the Scd galaxy, and NGC 4449 with the Irr galaxy - to create continuous spectral energy distributions. The fifth SED is a composite spectrum of Sa and Sab galaxies from Kinney et al. (1996). Our selection of these templates was motivated by our desire to use data of real galaxies, to avoid the uncertainties involved with spectra created artificially by stellar population models. This is also why we have not used interpolated galaxy spectra to create a finer mesh of SEDs.

The SEDs are presented in Figure 2. We will be using the term "galaxy spectral type" often in this paper; this simply means we are referring to galaxies with the same SEDs, and hence the same implied star formation rates, as those of the representative galaxies mentioned above.

We computed the colors of our template galaxies by first placing them onto a standard photometric system, to derive colors that could be used to test data on that system. Thus, we used the spectrophotometry of Hamuy et al. (1994), who have produced SEDs of a number of Southern Hemisphere standard stars across the UBVRI wavelength range. We convolved the SEDs of 14 of these stars with our filter and CCD response functions, to produce an "instrumental flux" through each filter, $I_{f}$ :

$$
I_{f}=\int T_{f}(\lambda) D(\lambda) F(\lambda) d \lambda
$$

where $T_{f}(\lambda)$ is the transmission of filter $f, D(\lambda)$ is the quantum efficiency of the CCD, and $F(\lambda)$ is the flux of the star. In other words, we were measuring a net flux from each star as if it had 
been observed through a telescope with a flat wavelength throughput of unity, with the exception of the filter and detector, and with no atmospheric extinction.

For the UBVR filters, we then used the photometric calibration of those stars by Landolt (1992) to run the PHOTCAL package in IRAF. Using this procedure, we obtained the photometric zero points and color terms with which we could transform any data simulated by our models onto the same standard UBVR system as HOGPW. The I75 and I86 filters are calibrated onto an absolute scale by HOGPW using the standard star Wolf 1346. To determine the transformation coefficients for these two filters, we processed the SED of Wolf 1346 through our procedure, and compared the instrumental flux with the absolute calibrated magnitudes defined in HOGPW to obtain the zero points. (Color terms were not used in the calibration of the I75 and I86 filters.)

The final derived parameters of the transformation from an "instrumental" magnitude to the HOGPW UBVRI75I86 photometric system, calibrated onto an absolute scale with Wolf 1346, is as follows:

$$
\begin{aligned}
& \mathrm{U}_{\text {inst }}=\mathrm{U}-17.79-0.042(\mathrm{U}-\mathrm{B}) \\
& \mathrm{B}_{\text {inst }}=\mathrm{B}-19.13-0.047(\mathrm{~B}-\mathrm{V}) \\
& \mathrm{V}_{\text {inst }}=\mathrm{V}-20.02-0.042(\mathrm{~V}-\mathrm{R}) \\
& \mathrm{R}_{\text {inst }}=\mathrm{R}-19.74-0.042(\mathrm{~V}-\mathrm{R}) \\
& \mathrm{I} 75_{\text {inst }}=\mathrm{I} 75-19.58 \\
& \mathrm{I} 86_{\text {inst }}=\mathrm{I} 86-19.26
\end{aligned}
$$

The formal errors for the transformation, as computed by PHOTCAL, are less than \pm 0.006 in magnitude and \pm 0.013 in the color term for each individual bandpass. Each model magnitude thus has an error of at most a few percent. The same is true for any color we choose to compute from these magnitudes.

The expected observed colors as the galaxies increase with redshift from $z=0$ to 1.0, in steps of $\Delta z=0.025$, were produced by applying K-corrections to the rest frame colors. The corrections were computed with direct numerical integration in the standard way:

$$
K_{f}=2.5 \log (1+z)+2.5 \log \frac{\int F(\lambda) T_{f}(\lambda) D(\lambda) d(\lambda)}{\int F[\lambda /(1+z)] T_{f}(\lambda) D(\lambda) d(\lambda)}
$$

We present our computed model galaxy colors for the five galaxy spectral types in Tables 1-5. To check our models, we compared them with the galaxy color indices from the large photometric surveys of Poulain \& Nieto (1994), Buta \& Williams (1995), and de Jong (1995). The template colors agree very well with median and mean color indices for the corresponding Hubble types, with at most \pm 0.05 magnitude variations. 


\section{The Photometric Redshift Method}

The photometric redshift method should, in principle, output both a redshift and a galaxy spectral type for each set of input from a galaxy. As demonstrated by Koo (1985), Connolly et al. (1995) and others, a combination of sufficient wavelength coverage (i.e. near-UV to near-IR) and data from at least four filters, such as $\mathrm{U} / \mathrm{B}_{J} / \mathrm{R}_{F} / \mathrm{I}_{N}$, is sufficient to obtain redshifts with roughly $z \pm 0.05$ accuracy. Thus the filter set we use here, which contains six filters and roughly the same wavelength coverage, is theoretically more than sufficient for the task of finding photometric redshifts. Additionally, the division of the Johnson I-band into two narrower bandpasses (I75 and I86) gives us additional leverage at higher redshifts, as prominent spectral features (especially the $4000 \AA$ break) are redshifted into that wavelength range.

As a first step, we need to know how well the various types of galaxies are separated in our multi-dimensional color space. In other words, how unique are the UBVRI75I86 colors of a galaxy with a given redshift and spectral type? A straightforward analysis shows that, in the $(\mathrm{U}-\mathrm{B}) /(\mathrm{B}-\mathrm{V}) /(\mathrm{V}-\mathrm{R}) /(\mathrm{R}-\mathrm{I} 75) /(\mathrm{I} 75-\mathrm{I} 86)$ color space, every galaxy redshift-spectral type pair on the previously stated binning interval is separated from every other such pair by at least 0.1 magnitude in at least one color; and the distinctions across broader wavelength ranges (e.g. B-R or R-I86) are even more pronounced.

The separation of SEDs in the multicolor space is most easily illustrated by tracing where the redshift-spectral type pairs lie on color-color diagrams. Figure 3 shows two color-color cuts in the multicolor space of the filter system, and the locations of the galaxy redshift-spectral type loci on them. These tracks can be thought of as color tracks for non-evolving galaxies as a function of redshift. In the (U-B)/(B-R) plane, galaxies with redshifts $z \leq 0.7$ are well separated. Similarly, in the $(\mathrm{B}-\mathrm{V}) /(\mathrm{R}-\mathrm{I} 86)$ plane, galaxies with $z \geq 0.4$ are well separated. A representative shift in the colors that would be created by extinction is shown with a reddening vector for $\mathrm{E}(\mathrm{B}-\mathrm{V})=0.1$.

These diagrams are also useful because they show the situations where the photometric redshift method is most and least effective. Early-type (E/S0 and Sab) galaxies are very easily separated from the other types, and move significantly in color space as a function of redshift; clearly, these galaxies are the ones most easily identified with this technique - a well-known fact that has been used by many authors (e.g. Im et al. 1996). Higher-redshift blue galaxies (especially irregulars), on the other hand, are somewhat problematic; increases in redshift around $0.4 \lesssim z \lesssim 0.6$, and near $z=1$, only slightly change the observed colors of these galaxy types. They are still unlikely to be confused with different galaxy spectral types; the intrinsic errors of their redshift determinations, however, will be larger. Some scatter can also come from variations in emission-line strength, particularly the $\mathrm{H} \alpha+\mathrm{N}[\mathrm{II}]$ complex for starburst galaxies; but the overall effect is unlikely to be more than 0.05-0.1 magnitude in any given color, which is less than the difference seen between almost all of the different redshift-type pairs on our grid of models. 


\section{Comparison with Spectroscopy}

The acid test of photometric redshifts is to compare them with spectroscopic redshifts. We used two distinct samples of galaxies, obtained independently and in different ways, and examined how well the photometric measurements match the spectroscopy separately and as a whole. This was done to see if our system yields the same results with different datasets obtained with this filter set, which is what we expect.

\subsection{Cluster Galaxy Data}

UBVRI75I86 photometry were obtained for four rich clusters of galaxies: Abell $963(z=0.20)$, CL1358+6245 $(z=0.32)$, CL3C295 $(z=0.46)$, and CL1601+4253 $(z=0.54)$. These clusters have numerous spectroscopic redshift measurements of cluster members of all spectral types, as well as some foreground and background galaxies (cf. Lavery \& Henry 1988; Fabricant, McClintock \& Bautz 1991; Dressler \& Gunn 1992), and a large range of redshifts, making them very desirable for testing the photometric redshift technique.

CCD imaging observations were made with the Steward Observatory 2.3-m telescope on Kitt Peak. The central $2^{\prime} \times 3^{\prime}$ of each cluster were imaged in UBV with a thinned, blue-sensitive $800 \times 1200 \mathrm{CCD}$, and the central $5^{\prime} \times 5^{\prime}$ were observed in RI75I86 with a $2048 \times 2048 \mathrm{CCD}$. Landolt (1992) broad-band standards and Massey et al. (1988) spectrophotometric standards were observed in every bandpass across all the airmass ranges observed for each night, and Wolf 1346 was always observed in RI75I86 to calibrate the nonstandard I-filters. The data were reduced and calibrated in the usual manner with IRAF; aperture photometry was then measured for the objects in those fields with published redshifts using APPHOT. We obtained photometry with 0.1 magnitude error or better in all six passbands for 42 of these galaxies.

\subsection{Field Galaxy Data}

Spectroscopic redshifts were also obtained for a number of galaxies in the HOGPW survey. 38 galaxies were measured using the Kitt Peak 4-meter telescope, in parallel with observations of quasar candidates in the survey fields (Hall et al. 1996b). We refer the reader to that work for the details of data acquisition and reduction. In summary, a number of observational setups were used: a single longslit, multislits, and the HYDRA multifiber positioner and bench spectrograph. 23 additional spectra were obtained with the Blue Channel Spectrograph on the Multiple Mirror Telescope. Again, the usual procedures in IRAF were used for data reduction and extraction of spectra. Redshifts were obtained by inspection of well known emission and absorption features (such as the Balmer lines, [OII] $\lambda 3727 \AA$, [OIII] $\lambda \lambda 4959,5007 \AA$, and the $4000 \AA$ break) or by cross-correlation using the XCOR task in IRAF. All 61 galaxies have UBVRI75I86 
photometry, which are calibrated with the procedures and parameters described in HOGPW.

\subsection{Photometric Redshifts}

Identifying the photometric redshift and spectral type of any given galaxy involves finding its best matching redshift-type template. Clearly, the simplest definition of a match is that the template SED matches the data more closely than any other template. This is rarely a trivial criterion to meet, however, since every real galaxy is at least slightly different from any template galaxy. In each color, there is a difference between a template SED's color and the observed color.

One straightforward scheme to determine the closest match between an observed galaxy and a template would be some kind of least-squares method - comparing the observed flux in each waveband with the flux predicted by a template. We tested several variations of this method, but it proved not to be optimal for the purposes of our study. One reason is that we want to identify the redshift and spectral type of a galaxy without any knowledge of its apparent magnitude. Thus we must use the comparison of galaxy colors, rather than passband fluxes. Also, our tests showed that a least-squares technique can estimate the redshift of a galaxy reliably, but too often misclassifies its spectral type.

A more successful approach, which we ultimately adopted, was to look for matches by seeking the smallest maximum difference between the observed and template colors. (Such a method might loosely be compared with a least-squares method the way a Kolmogorov-Smirnov test might compare with a $\chi^{2}$-test, when measuring the quality of a model fitted to data.) This kind of strategy yielded the best results in predicting both the redshift and spectral type of a galaxy, independent of its apparent magnitude. Examination of the color distributions of the model SEDs, combined with empirical tests of choosing matches using various procedures, led us to the following algorithm for selecting matches most accurately:

$<\mathrm{i}>$ Each model-vs.-data comparison yields a set of $\Delta_{i-j} \equiv(i-j)_{\text {galaxy }}-(i-j)_{\text {model }}$, where $i-j=\mathrm{U}-\mathrm{B}, \mathrm{B}-\mathrm{V}, \mathrm{V}-\mathrm{R}, \mathrm{R}-\mathrm{I} 75, \mathrm{I} 75-\mathrm{I} 86$. The most likely match is the comparison that yields the minimum largest $\Delta_{i-j}$. The value of such a maximum for an accurately matched redshift-type pair is typically $\Delta_{i-j} \max \sim 0.1$ to 0.2 magnitude.

$<$ ii $>$ If $\Delta_{i-j} \max$ is large (i.e. $>0.2$ ) for all possible matches, the comparisons that yield the minimum second - largest $\Delta_{i-j}$ are also reviewed. This step takes into account the possibility that photometry for that galaxy may have been anomalously affected in one filter, perhaps by an emission line or other spectral feature. Comparisons which yield very large values of $\Delta_{i-j} \max$ $(>0.5)$, however, are not considered possible matches.

$<$ iii $>$ If two or more matches have similar $\Delta_{i-j} \max$, the best match is usually the one where the sum of $\Delta_{i-j}$ 's is closest to zero. Matches with similar $\Delta_{i-j}$ characteristics are almost always of the same galaxy spectral type, with slightly different redshifts; in cases where it appears $\Sigma \Delta_{i-j}$ 
would be closest to zero in an interpolation between two adjacent redshift steps, the midpoint between those two steps is designated the most likely redshift.

$<$ iv $>$ Finally, if two matches meet the above criteria with the same accuracy, the one most closely matching in (U-B) or (I75-I86), for objects with likely redshift less than or greater than 0.5 respectively, is designated the more likely match. This condition is based on the fact that the separations in $F_{\lambda}$ of galaxy SEDs are widest in the UV and far-red wavelengths (see Figure 1).

\section{Discussion}

A computer program called "GetZ" was written which automates the above analysis. The colors of the 103 sample galaxies were then run through the program. We present in Figure 4 a direct comparison of $z_{p}$, the photometrically determined redshifts output by "GetZ," with the

spectroscopically measured redshifts $z_{s}$. The diagonal line is not a fit to the data, but the locus of exactly perfect correspondence (i.e. $z_{s}=z_{p}$ ). The dispersion measure $z_{p}-z_{s}$ for the entire sample is plotted in histogram form in Figure 5.

The rms deviation of all the galaxies in the sample, $\sigma_{z}$, is $0.053 .87 \%$ of the galaxies have $\left|z_{p}-z_{s}\right|<0.1$. As Figure 5 shows, the distribution of $z_{p}-z_{s}$ is essentially symmetric about $z_{p}-z_{s}=0$; in fact, $\Sigma\left(z_{p}-z_{s}\right)=0.002$, and the error distribution is consistent with a Gaussian distribution. These results are consistent with the accuracy we expected, and with those in the literature.

\subsection{The Effects of "E+A" Galaxies}

The cluster galaxy subsample had a slightly higher $\sigma_{z}$ than the HOGPW subsample $(0.060$ vs. 0.046). Statistically, the lower accuracy of the cluster sample is barely significant, but it is instructive to examine the cause of this slight discrepancy.

The cluster samples were all taken near the centers of rich clusters of galaxies which exhibit the so-called Butcher-Oemler effect (Butcher \& Oemler 1978). Not surprisingly, a number of post-starburst, or "E+A" galaxies (Dressler \& Gunn 1983; Couch \& Sharples 1987; Liu \& Green 1996), were observed in the CCD image fields which we used to obtain photometry of the sample galaxies. E+A galaxies have a spectrum characterized by strong Balmer absorption, weak or no line emission, and the earmarks of an old stellar population. Their spectral energy distributions are thus a hybrid, typically with colors bluer than ellipticals but redder than late-type spirals. A priori, then, it seems plausible that E+A galaxies could systematically confuse the redshift-spectral type comparison scheme, since their colors are neither truly spiral nor truly elliptical.

To test this hypothesis, we have selected the E+A galaxies in the cluster sample - those 
objected which were designated "a" or "A" by Dressler \& Gunn (1992), or "E+A" by Fabricant et al. (1991) - and plotted their $z_{p}-z_{s}$ distributions separately (with shaded bars) on Figure 5. The E+A's are clearly less accurately identified than the other galaxy types. $\sigma_{z}$ for the E+A's is 0.097 for 13 objects; and it is half that value ( 0.047 for 29 objects) for the rest of the cluster sample if the E+A's are excluded. Figure 5 shows also that the $z_{p}$ 's which are the worst underestimates of the true redshifts (i.e. the four objects where $z_{p}-z_{s}<-0.12$ ) are all $\mathrm{E}+\mathrm{A}$ galaxies. Although about half $(6 / 13)$ of the E+A's were successfully measured to within 0.05 of their spectroscopic redshifts, our results demonstrate that $\mathrm{E}+\mathrm{A}$ galaxies can increase the uncertainty of photometric redshift measurements and cause systematic underestimates of $z_{p}$.

Can this problem be overcome by simply creating one or more templates of $\mathrm{E}+\mathrm{A}$ galaxies and adding them to the photometric redshift algorithm? Unfortunately, this is not a solution. As shown by Liu \& Green (1996), E+A galaxies are a rather heterogeneous population which cannot be characterized by one or even a few templates. Their SEDs vary widely depending on the strength of the decaying starburst, and on the underlying old stellar population. Furthermore, no UV spectrophotometry of $\mathrm{E}+\mathrm{A}$ galaxies exist in the literature; $\mathrm{E}+\mathrm{A}$ templates can therefore be produced only by stellar population models, which we have explicitly avoided in this work. Since E+A galaxies appear to be rare in the field, however (Zabludoff et al. 1996), the random error contributed by E+A's is likely to be negligible in field galaxy studies with photometric redshifts.

\subsection{Identifications With Incomplete Data}

In any galaxy survey where a photometric redshift scheme such as ours is likely to be applied, some portion of the galaxies will have incomplete color information, such as a non-detection in one or more filters. This is especially likely for faint early-type galaxies; as Table 1 shows, if an unevolved elliptical is detected in $\mathrm{R}$ at a given apparent magnitude, the survey data must extend at least two magnitudes fainter in $U$ to be detected if the galaxy is at $z=0$, and four magnitudes if it is at $\mathrm{z}=0.4$. It is important to know if redshift identifications are still accurate or possible with missing information, particularly U-band.

We can get some idea of what results to expect with color-color diagrams. From Figure 1, it is clear that lack of U-band data does not significantly affect the separation of high-redshift galaxies from each other; the problem with losing blue and UV data lies in confusing low-redshift, redder galaxies from higher-redshift bluer galaxies. If we exclude U-band data and use all the photometry from B redward (see Figure 6), the colors of E/S0 and Sab galaxies from $0 \lesssim \mathrm{z} \lesssim 0.25$ are essentially degenerate with those of Sbc galaxies from $0.1 \lesssim \mathrm{z} \lesssim 0.4$ and Scd galaxies from $0.2 \lesssim \mathrm{z} \lesssim 0.6$. If both $\mathrm{U}$ and $\mathrm{B}$ data are missing, the risk of low-redshift confusion is even greater.

Empirical tests confirm the problems with measuring photometric redshifts which are suggested by the color-color plots in Figure 6. The BVRI75I86 colors for the sample galaxies were input into the "GetZ" program, and $z_{p}$ was determined for each object as before. This time, $\sigma_{z}=$ 
0.101, double the value of $\sigma_{z}$ computed with U-band data. Furthermore, galaxies which were identified as spiral or irregular with $\mathrm{z}<0.4$ had $\sigma_{z}=0.160$; the scatter is much larger, and there is a systematic tendency to misidentify Scd and Irr galaxies near z 0.3 as Sbc galaxies near z 0.05.

On the other hand, $\sigma_{z}$ for all galaxies with $\mathrm{z}>0.4$ and for early-type galaxies with $\mathrm{z}>0.25$ were 0.053 and 0.057 respectively. Apparently there is little reduction in the typical accuracies of $z_{p}$ for those subsamples despite the lack of U-band data; this is also predicted by the color-color plots, and is the fortuitous result of the $4000 \AA$ break being redshifted into the $\mathrm{V}$ and $\mathrm{R}$ bands, away from the U-band. If it were possible, then, to select galaxies which are definitely early-type or at high redshift, our photometric method can still be effective for determining redshifts, even without the U-band data which is so critical for measuring later-type galaxies at lower redshifts.

\section{Conclusions}

We have shown that our photometric redshift method based on the broad-band colors UBVR, I75 and I86 can determine redshifts to a typical accuracy of $\mathrm{z}= \pm 0.05$ for field and cluster galaxies, and approximate their spectral types as well. This result extends the increasing amount of literature that confirms the validity of using multicolor broad-band photometry to obtain a redshift distribution for samples of galaxies. Since our system relies on colors alone, it is somewhat more versatile than systems which are dependent on other galaxy parameters such as apparent magnitude. Thus, it can be (and has been - see Liu et al. 1998) applied to a wider range of astrophysical problems, such as field galaxy evolution as a function of redshift.

It should be emphasized that any photometric redshift system is most effectively used as a statistical tool for measuring the redshift distribution of a galaxy sample, rather than for assigning unambiguous redshifts to individual galaxies. Just as a significant fraction of galaxies defy straightforward classification on the morphological Hubble sequence, galaxies which are not spectrophotometrically "normal" - such as E+A galaxies - can cause systematic errors in redshift and spectral type determinations. Incomplete data, such as a lack of U-band photometry, can also produce serious mistakes in $z_{p}$ measurements; this can be overcome, however, by selecting samples of higher-redshift and/or early-type galaxies for study. With a healthy awareness of the method's strengths and weaknesses, and a careful attention to detail, using multicolors to obtain galaxy redshifts and spectral types is a feasible and powerful technique for use in the study of galaxy populations.

We thank Pat Hall and Pat Osmer for invaluable assistance in obtaining much of the spectroscopic comparison data, and Andy Connolly and David Koo for helpful discussions. C. L. gratefully acknowledges support from NASA grant NGT-50758. 


\section{REFERENCES}

Baum, W. A. 1962, Problems of Extragalactic Research, IAU Symposium No. 15 (MacMillan, New York), 390

Brunner, R. J., Connolly, A. C., Szalay, A. S., \& Bershady, M. A. 1997, ApJ, 482, L21.

Buta, R., \& Williams, K. L. 1995, AJ, 109, 543

Butcher, H., \& Oemler, A., Jr. 1978, ApJ, 219, 18.

Coleman, G. D., Wu, C.-C., \& Weedman, D. W. 1980, ApJS, 43, 393

Connolly, A. J., Csabai, I., Szalay, A. S., Koo, D. C., Kron, R. C., \& Munn, J. A. 1995, AJ, 110, 2655

Couch, W. J., \& Sharples, R. M. 1987, MNRAS, 229, 423

Dressler, A. \& Gunn, J. E. 1983, ApJ, 270, 7

Dressler, A. \& Gunn, J. E. 1992, ApJS, 78, 1

Fabricant, D., McClintock, J., \& Bautz, M. 1991, ApJ, 381, 33

Hall, P. B., Osmer, P. S., Green, R. F., Porter, A. C., \& Warren, S. J. 1996a (HOGPW), ApJS, 104,185

Hall, P. B., Osmer, P. S., Green, R. F., Porter, A. C., \& Warren, S. J. 1996b, ApJ, 462, 614

Hamuy, M., Suntzeff, N., Heathcote, S., Walker, A., Gigoux, P, \& Phillips, M. 1996, PASP, 106, 566

Im, M., Griffiths, R., Ratnatunga, K., \& Sarajedini, V. L. 1996, ApJ, 461, L79

de Jong, R. S. 1995, Ph. D. Thesis, University of Groningen.

Kennicutt, R. C. 1992, ApJS, 79, 255

Kinney, A. L., Calzetti, D., Bohlin, R., McQuade, K., Storchi-Bergman, T., \& Schmitt, H. R. 1996, ApJ, 467, 38.

Koo, D. C. 1985, AJ, 90, 418

Landolt, A. U. 1992, AJ, 104, 372

Lavery, R. \& Henry, J. 1988, ApJ, 330, 596

Liu, C. T. 1996, Ph. D. Thesis, University of Arizona

Liu, C. T., \& Green, R. F. 1996, ApJ, 458, L63

Liu, C. T., Green, R. F., Hall, P. B., \& Osmer, P. S. 1998, AJ, submitted

Loh, E. D., \& Spillar, E. J. 1986, ApJ, 303, 154

Massey, P., Strobel, K, Barnes, J. V., \& Anderson, E. 1988, ApJ, 328, 315

Poulain, P. \& Nieto, J.-L. 1994, A\&AS, 103, 573 
Sawicki, M. J., Lin, H., \& Yee, H. K.-C. 1997, AJ, 113, 1.

Zabludoff, A. I., Zaritsky, D., Lin, H., Tucker, D., Hashimoto, Y., Shectman, S., Oemler, A., \& Kirshner, R. P. 1996, ApJ, 466, 104. 
Table 1. E/S0 template galaxy colors

\begin{tabular}{cccccc}
\hline \hline $\mathrm{z}$ & $\mathrm{U}-\mathrm{B}$ & $\mathrm{B}-\mathrm{V}$ & $\mathrm{V}-\mathrm{R}$ & $\mathrm{R}-\mathrm{I} 75$ & $\mathrm{I} 75-\mathrm{I} 86$ \\
\hline 0.000 & 0.539 & 0.924 & 0.590 & 0.143 & 0.243 \\
0.025 & 0.490 & 1.016 & 0.604 & 0.150 & 0.229 \\
0.050 & 0.436 & 1.115 & 0.613 & 0.154 & 0.226 \\
0.075 & 0.393 & 1.216 & 0.627 & 0.161 & 0.228 \\
0.100 & 0.362 & 1.310 & 0.643 & 0.180 & 0.230 \\
0.125 & 0.342 & 1.389 & 0.668 & 0.184 & 0.260 \\
0.150 & 0.341 & 1.452 & 0.699 & 0.194 & 0.280 \\
0.175 & 0.360 & 1.503 & 0.728 & 0.221 & 0.278 \\
0.200 & 0.406 & 1.543 & 0.761 & 0.240 & 0.279 \\
0.225 & 0.480 & 1.568 & 0.806 & 0.250 & 0.284 \\
0.250 & 0.572 & 1.565 & 0.874 & 0.257 & 0.299 \\
0.275 & 0.668 & 1.539 & 0.964 & 0.257 & 0.315 \\
0.300 & 0.765 & 1.506 & 1.059 & 0.251 & 0.330 \\
0.325 & 0.867 & 1.478 & 1.145 & 0.256 & 0.335 \\
0.350 & 0.969 & 1.456 & 1.223 & 0.265 & 0.346 \\
0.375 & 1.058 & 1.439 & 1.292 & 0.270 & 0.373 \\
0.400 & 1.122 & 1.433 & 1.347 & 0.282 & 0.403 \\
0.425 & 1.155 & 1.441 & 1.387 & 0.311 & 0.423 \\
0.450 & 1.153 & 1.461 & 1.418 & 0.354 & 0.428 \\
0.475 & 1.114 & 1.495 & 1.441 & 0.398 & 0.428 \\
0.500 & 1.039 & 1.546 & 1.453 & 0.454 & 0.415 \\
0.525 & 0.935 & 1.611 & 1.444 & 0.526 & 0.404 \\
0.550 & 0.812 & 1.685 & 1.409 & 0.607 & 0.403 \\
0.575 & 0.680 & 1.760 & 1.364 & 0.685 & 0.408 \\
0.600 & 0.544 & 1.833 & 1.322 & 0.749 & 0.417 \\
0.625 & 0.412 & 1.902 & 1.291 & 0.786 & 0.439 \\
0.650 & 0.287 & 1.968 & 1.267 & 0.801 & 0.480 \\
0.675 & 0.174 & 2.025 & 1.247 & 0.810 & 0.531 \\
0.700 & 0.075 & 2.070 & 1.233 & 0.811 & 0.597 \\
0.725 & -0.009 & 2.101 & 1.228 & 0.809 & 0.673 \\
0.750 & -0.077 & 2.116 & 1.235 & 0.806 & 0.749 \\
0.775 & -0.131 & 2.115 & 1.253 & 0.798 & 0.820 \\
0.800 & -0.170 & 2.097 & 1.281 & 0.772 & 0.895 \\
0.825 & -0.194 & 2.060 & 1.321 & 0.726 & 0.975 \\
0.850 & -0.207 & 2.007 & 1.371 & 0.663 & 1.055 \\
0.875 & -0.209 & 1.936 & 1.432 & 0.599 & 1.122 \\
0.900 & -0.206 & 1.854 & 1.499 & 0.537 & 1.173 \\
0.925 & -0.200 & 1.763 & 1.565 & 0.478 & 1.218 \\
0.950 & -0.191 & 1.664 & 1.629 & 0.431 & 1.247 \\
0.975 & -0.184 & 1.563 & 1.689 & 0.418 & 1.243 \\
1.000 & -0.178 & 1.459 & 1.749 & 0.437 & 1.209 \\
\hline & & & & &
\end{tabular}


Table 2. Sab template galaxy colors

\begin{tabular}{cccccc}
\hline \hline $\mathrm{z}$ & $\mathrm{U}-\mathrm{B}$ & $\mathrm{B}-\mathrm{V}$ & $\mathrm{V}-\mathrm{R}$ & $\mathrm{R}-\mathrm{I} 75$ & $\mathrm{I} 75-\mathrm{I} 86$ \\
\hline 0.000 & 0.364 & 0.853 & 0.595 & 0.173 & 0.252 \\
0.025 & 0.385 & 0.903 & 0.617 & 0.173 & 0.260 \\
0.050 & 0.379 & 0.966 & 0.634 & 0.169 & 0.285 \\
0.075 & 0.354 & 1.039 & 0.646 & 0.177 & 0.303 \\
0.100 & 0.316 & 1.114 & 0.654 & 0.205 & 0.295 \\
0.125 & 0.275 & 1.188 & 0.662 & 0.215 & 0.307 \\
0.150 & 0.241 & 1.259 & 0.671 & 0.230 & 0.309 \\
0.175 & 0.218 & 1.328 & 0.676 & 0.257 & 0.297 \\
0.200 & 0.206 & 1.390 & 0.684 & 0.269 & 0.299 \\
0.225 & 0.207 & 1.446 & 0.696 & 0.289 & 0.296 \\
0.250 & 0.226 & 1.480 & 0.725 & 0.306 & 0.309 \\
0.275 & 0.257 & 1.496 & 0.769 & 0.310 & 0.333 \\
0.300 & 0.287 & 1.500 & 0.823 & 0.300 & 0.354 \\
0.325 & 0.312 & 1.496 & 0.882 & 0.295 & 0.366 \\
0.350 & 0.333 & 1.479 & 0.946 & 0.285 & 0.387 \\
0.375 & 0.343 & 1.453 & 1.011 & 0.273 & 0.413 \\
0.400 & 0.338 & 1.425 & 1.074 & 0.271 & 0.439 \\
0.425 & 0.327 & 1.398 & 1.134 & 0.277 & 0.460 \\
0.450 & 0.316 & 1.374 & 1.192 & 0.289 & 0.474 \\
0.475 & 0.302 & 1.356 & 1.244 & 0.302 & 0.481 \\
0.500 & 0.279 & 1.346 & 1.291 & 0.326 & 0.472 \\
0.525 & 0.240 & 1.343 & 1.324 & 0.368 & 0.457 \\
0.550 & 0.188 & 1.351 & 1.335 & 0.420 & 0.440 \\
0.575 & 0.129 & 1.371 & 1.327 & 0.472 & 0.426 \\
0.600 & 0.067 & 1.393 & 1.311 & 0.524 & 0.413 \\
0.625 & 0.001 & 1.415 & 1.293 & 0.566 & 0.412 \\
0.650 & -0.065 & 1.433 & 1.271 & 0.602 & 0.425 \\
0.675 & -0.126 & 1.448 & 1.240 & 0.643 & 0.439 \\
0.700 & -0.181 & 1.457 & 1.205 & 0.679 & 0.465 \\
0.725 & -0.232 & 1.454 & 1.176 & 0.716 & 0.497 \\
0.750 & -0.279 & 1.440 & 1.157 & 0.751 & 0.534 \\
0.775 & -0.321 & 1.418 & 1.146 & 0.782 & 0.570 \\
0.800 & -0.355 & 1.393 & 1.137 & 0.799 & 0.616 \\
0.825 & -0.382 & 1.366 & 1.132 & 0.796 & 0.672 \\
0.850 & -0.404 & 1.337 & 1.128 & 0.776 & 0.738 \\
0.875 & -0.419 & 1.297 & 1.134 & 0.749 & 0.799 \\
0.900 & -0.429 & 1.252 & 1.150 & 0.721 & 0.848 \\
0.925 & -0.433 & 1.205 & 1.177 & 0.668 & 0.906 \\
0.950 & -0.431 & 1.155 & 1.209 & 0.596 & 0.974 \\
0.975 & -0.428 & 1.107 & 1.237 & 0.548 & 1.018 \\
1.000 & -0.427 & 1.062 & 1.255 & 0.510 & 1.057 \\
\hline & & & & &
\end{tabular}


Table 3. Sbc template galaxy colors

\begin{tabular}{cccccc}
\hline \hline $\mathrm{z}$ & $\mathrm{U}-\mathrm{B}$ & $\mathrm{B}-\mathrm{V}$ & $\mathrm{V}-\mathrm{R}$ & $\mathrm{R}-\mathrm{I} 75$ & $\mathrm{I} 75-\mathrm{I} 86$ \\
\hline 0.000 & 0.055 & 0.661 & 0.487 & 0.074 & 0.273 \\
0.025 & 0.103 & 0.705 & 0.499 & 0.068 & 0.272 \\
0.050 & 0.142 & 0.758 & 0.510 & 0.065 & 0.267 \\
0.075 & 0.167 & 0.818 & 0.520 & 0.086 & 0.239 \\
0.100 & 0.178 & 0.880 & 0.528 & 0.118 & 0.205 \\
0.125 & 0.178 & 0.941 & 0.539 & 0.123 & 0.199 \\
0.150 & 0.167 & 1.000 & 0.549 & 0.130 & 0.198 \\
0.175 & 0.141 & 1.064 & 0.549 & 0.147 & 0.195 \\
0.200 & 0.105 & 1.127 & 0.553 & 0.132 & 0.222 \\
0.225 & 0.060 & 1.184 & 0.567 & 0.145 & 0.219 \\
0.250 & 0.010 & 1.228 & 0.596 & 0.167 & 0.224 \\
0.275 & -0.044 & 1.263 & 0.633 & 0.179 & 0.252 \\
0.300 & -0.097 & 1.289 & 0.675 & 0.177 & 0.271 \\
0.325 & -0.148 & 1.307 & 0.720 & 0.175 & 0.276 \\
0.350 & -0.196 & 1.313 & 0.770 & 0.165 & 0.277 \\
0.375 & -0.241 & 1.308 & 0.819 & 0.153 & 0.276 \\
0.400 & -0.283 & 1.298 & 0.861 & 0.149 & 0.295 \\
0.425 & -0.319 & 1.280 & 0.904 & 0.154 & 0.319 \\
0.450 & -0.351 & 1.254 & 0.952 & 0.163 & 0.341 \\
0.475 & -0.378 & 1.221 & 0.999 & 0.177 & 0.350 \\
0.500 & -0.401 & 1.182 & 1.045 & 0.205 & 0.343 \\
0.525 & -0.421 & 1.139 & 1.082 & 0.246 & 0.330 \\
0.550 & -0.439 & 1.093 & 1.111 & 0.289 & 0.317 \\
0.575 & -0.455 & 1.046 & 1.134 & 0.329 & 0.305 \\
0.600 & -0.468 & 0.998 & 1.155 & 0.361 & 0.293 \\
0.625 & -0.480 & 0.950 & 1.174 & 0.383 & 0.292 \\
0.650 & -0.492 & 0.905 & 1.183 & 0.404 & 0.302 \\
0.675 & -0.504 & 0.862 & 1.182 & 0.431 & 0.318 \\
0.700 & -0.513 & 0.821 & 1.176 & 0.460 & 0.340 \\
0.725 & -0.522 & 0.782 & 1.169 & 0.487 & 0.371 \\
0.750 & -0.530 & 0.747 & 1.156 & 0.513 & 0.408 \\
0.775 & -0.536 & 0.715 & 1.139 & 0.537 & 0.445 \\
0.800 & -0.540 & 0.686 & 1.118 & 0.560 & 0.479 \\
0.825 & -0.543 & 0.660 & 1.092 & 0.579 & 0.515 \\
0.850 & -0.544 & 0.638 & 1.062 & 0.588 & 0.557 \\
0.875 & -0.542 & 0.617 & 1.030 & 0.588 & 0.599 \\
0.900 & -0.538 & 0.599 & 0.996 & 0.596 & 0.626 \\
0.925 & -0.532 & 0.583 & 0.961 & 0.595 & 0.654 \\
0.950 & -0.524 & 0.569 & 0.925 & 0.580 & 0.693 \\
0.975 & -0.513 & 0.556 & 0.890 & 0.575 & 0.721 \\
1.000 & -0.501 & 0.545 & 0.855 & 0.569 & 0.751 \\
\hline & & & & &
\end{tabular}


Table 4. Scd template galaxy colors

\begin{tabular}{cccccc}
\hline \hline $\mathrm{z}$ & $\mathrm{U}-\mathrm{B}$ & $\mathrm{B}-\mathrm{V}$ & $\mathrm{V}-\mathrm{R}$ & $\mathrm{R}-\mathrm{I} 75$ & $\mathrm{I} 75-\mathrm{I} 86$ \\
\hline 0.000 & -0.077 & 0.601 & 0.465 & -0.018 & 0.144 \\
0.025 & -0.048 & 0.630 & 0.485 & -0.014 & 0.151 \\
0.050 & -0.039 & 0.669 & 0.500 & -0.005 & 0.154 \\
0.075 & -0.051 & 0.718 & 0.508 & 0.044 & 0.119 \\
0.100 & -0.077 & 0.769 & 0.513 & 0.106 & 0.073 \\
0.125 & -0.112 & 0.821 & 0.517 & 0.126 & 0.071 \\
0.150 & -0.149 & 0.872 & 0.520 & 0.143 & 0.076 \\
0.175 & -0.188 & 0.928 & 0.509 & 0.162 & 0.087 \\
0.200 & -0.226 & 0.981 & 0.498 & 0.134 & 0.142 \\
0.225 & -0.264 & 1.030 & 0.494 & 0.148 & 0.154 \\
0.250 & -0.299 & 1.061 & 0.505 & 0.170 & 0.186 \\
0.275 & -0.331 & 1.079 & 0.528 & 0.182 & 0.243 \\
0.300 & -0.361 & 1.085 & 0.561 & 0.178 & 0.281 \\
0.325 & -0.386 & 1.081 & 0.598 & 0.172 & 0.299 \\
0.350 & -0.411 & 1.060 & 0.645 & 0.158 & 0.302 \\
0.375 & -0.436 & 1.031 & 0.693 & 0.142 & 0.286 \\
0.400 & -0.461 & 0.997 & 0.738 & 0.128 & 0.300 \\
0.425 & -0.488 & 0.961 & 0.784 & 0.118 & 0.328 \\
0.450 & -0.514 & 0.922 & 0.833 & 0.112 & 0.351 \\
0.475 & -0.541 & 0.883 & 0.878 & 0.112 & 0.362 \\
0.500 & -0.568 & 0.845 & 0.921 & 0.123 & 0.358 \\
0.525 & -0.595 & 0.808 & 0.951 & 0.153 & 0.342 \\
0.550 & -0.621 & 0.773 & 0.968 & 0.193 & 0.320 \\
0.575 & -0.645 & 0.741 & 0.975 & 0.229 & 0.303 \\
0.600 & -0.668 & 0.710 & 0.975 & 0.264 & 0.284 \\
0.625 & -0.688 & 0.681 & 0.971 & 0.291 & 0.269 \\
0.650 & -0.708 & 0.655 & 0.953 & 0.322 & 0.263 \\
0.675 & -0.724 & 0.629 & 0.925 & 0.358 & 0.263 \\
0.700 & -0.739 & 0.604 & 0.894 & 0.396 & 0.268 \\
0.725 & -0.751 & 0.579 & 0.863 & 0.434 & 0.280 \\
0.750 & -0.761 & 0.554 & 0.834 & 0.467 & 0.302 \\
0.775 & -0.768 & 0.529 & 0.806 & 0.496 & 0.328 \\
0.800 & -0.772 & 0.503 & 0.779 & 0.519 & 0.356 \\
0.825 & -0.773 & 0.477 & 0.755 & 0.528 & 0.392 \\
0.850 & -0.772 & 0.452 & 0.731 & 0.524 & 0.435 \\
0.875 & -0.768 & 0.426 & 0.710 & 0.509 & 0.480 \\
0.900 & -0.761 & 0.401 & 0.691 & 0.495 & 0.513 \\
0.925 & -0.752 & 0.377 & 0.674 & 0.467 & 0.553 \\
0.950 & -0.742 & 0.355 & 0.657 & 0.423 & 0.606 \\
0.975 & -0.730 & 0.334 & 0.642 & 0.387 & 0.651 \\
1.000 & -0.716 & 0.315 & 0.626 & 0.353 & 0.695 \\
\hline & & & & &
\end{tabular}


Table 5. Irr template galaxy colors

\begin{tabular}{cccccc}
\hline \hline $\mathrm{z}$ & $\mathrm{U}-\mathrm{B}$ & $\mathrm{B}-\mathrm{V}$ & $\mathrm{V}-\mathrm{R}$ & $\mathrm{R}-\mathrm{I} 75$ & $\mathrm{I} 75-\mathrm{I} 86$ \\
\hline 0.000 & -0.419 & 0.274 & 0.335 & -0.090 & 0.191 \\
0.025 & -0.376 & 0.311 & 0.314 & -0.092 & 0.188 \\
0.050 & -0.341 & 0.349 & 0.300 & -0.079 & 0.170 \\
0.075 & -0.326 & 0.392 & 0.285 & -0.009 & 0.096 \\
0.100 & -0.341 & 0.438 & 0.269 & 0.072 & 0.014 \\
0.125 & -0.369 & 0.484 & 0.256 & 0.086 & 0.000 \\
0.150 & -0.405 & 0.530 & 0.248 & 0.084 & 0.002 \\
0.175 & -0.445 & 0.580 & 0.235 & 0.074 & 0.015 \\
0.200 & -0.487 & 0.624 & 0.252 & -0.030 & 0.096 \\
0.225 & -0.531 & 0.668 & 0.290 & -0.069 & 0.106 \\
0.250 & -0.575 & 0.700 & 0.319 & -0.078 & 0.153 \\
0.275 & -0.619 & 0.726 & 0.340 & -0.077 & 0.225 \\
0.300 & -0.659 & 0.745 & 0.360 & -0.082 & 0.257 \\
0.325 & -0.692 & 0.773 & 0.364 & -0.092 & 0.259 \\
0.350 & -0.718 & 0.766 & 0.393 & -0.103 & 0.229 \\
0.375 & -0.741 & 0.741 & 0.433 & -0.111 & 0.162 \\
0.400 & -0.758 & 0.709 & 0.471 & -0.099 & 0.127 \\
0.425 & -0.773 & 0.674 & 0.511 & -0.054 & 0.088 \\
0.450 & -0.784 & 0.634 & 0.555 & -0.012 & 0.054 \\
0.475 & -0.794 & 0.592 & 0.598 & 0.007 & 0.040 \\
0.500 & -0.801 & 0.549 & 0.640 & 0.015 & 0.033 \\
0.525 & -0.809 & 0.507 & 0.674 & 0.040 & 0.011 \\
0.550 & -0.815 & 0.466 & 0.700 & 0.049 & 0.008 \\
0.575 & -0.819 & 0.426 & 0.720 & 0.032 & 0.034 \\
0.600 & -0.822 & 0.389 & 0.738 & 0.035 & 0.038 \\
0.625 & -0.823 & 0.355 & 0.770 & 0.026 & 0.048 \\
0.650 & -0.824 & 0.326 & 0.773 & 0.046 & 0.077 \\
0.675 & -0.825 & 0.301 & 0.754 & 0.084 & 0.124 \\
0.700 & -0.825 & 0.281 & 0.724 & 0.130 & 0.153 \\
0.725 & -0.823 & 0.263 & 0.692 & 0.174 & 0.175 \\
0.750 & -0.822 & 0.249 & 0.660 & 0.213 & 0.191 \\
0.775 & -0.819 & 0.236 & 0.627 & 0.249 & 0.191 \\
0.800 & -0.816 & 0.226 & 0.593 & 0.277 & 0.175 \\
0.825 & -0.812 & 0.217 & 0.560 & 0.300 & 0.171 \\
0.850 & -0.807 & 0.209 & 0.528 & 0.311 & 0.185 \\
0.875 & -0.802 & 0.203 & 0.497 & 0.321 & 0.203 \\
0.900 & -0.796 & 0.198 & 0.468 & 0.344 & 0.207 \\
0.925 & -0.790 & 0.194 & 0.443 & 0.353 & 0.219 \\
0.950 & -0.782 & 0.190 & 0.418 & 0.338 & 0.254 \\
0.975 & -0.775 & 0.188 & 0.396 & 0.310 & 0.302 \\
1.000 & -0.768 & 0.188 & 0.375 & 0.271 & 0.358 \\
\hline & & & & &
\end{tabular}


Fig. 1 Filter transmissions and CCD quantum efficiency vs. wavelength.

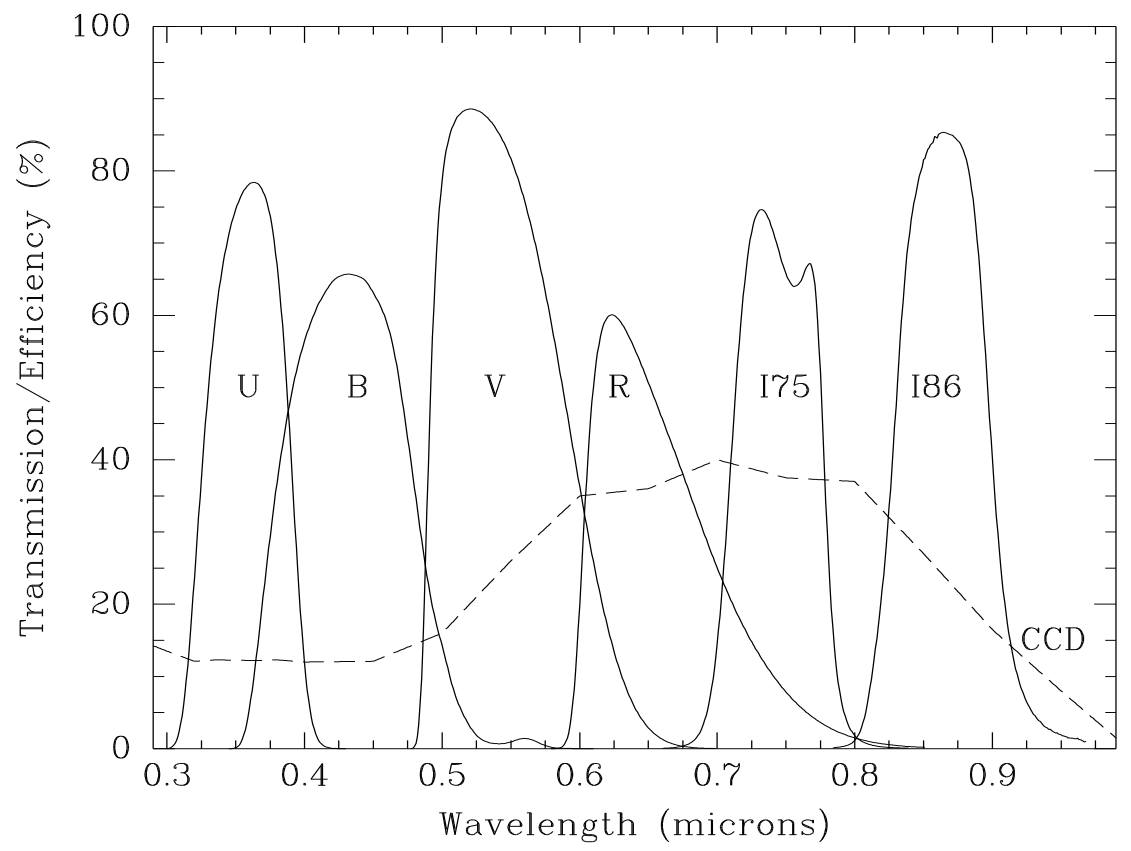


Fig. 2 Spectral energy distributions of the template galaxies, in units of $F_{\lambda}$ normalized to unity at $5500 \AA$.

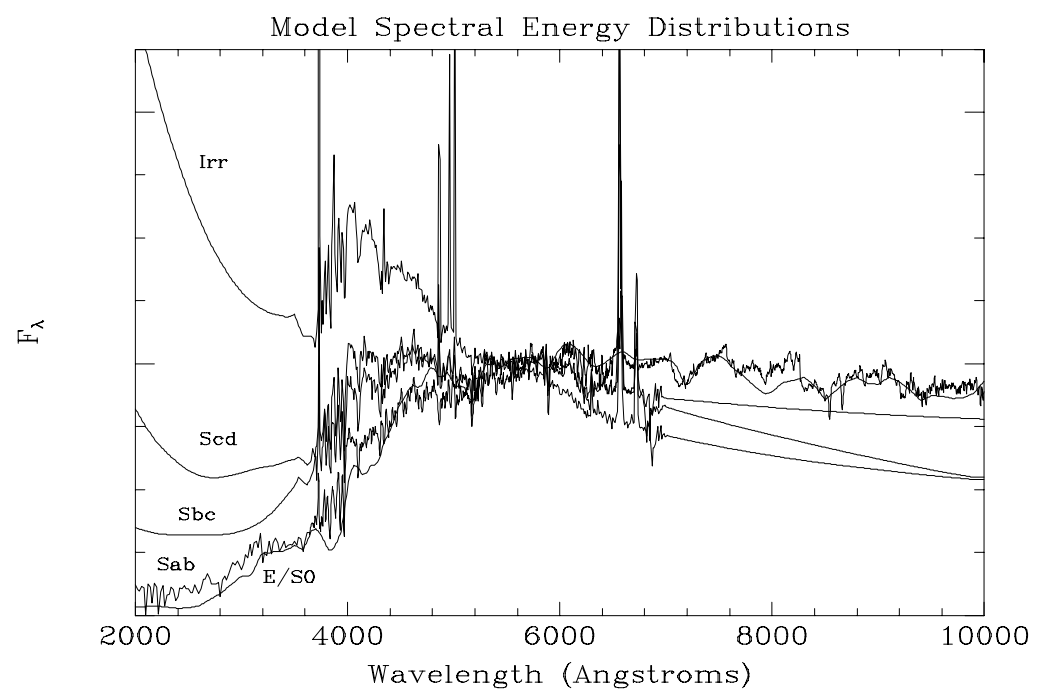


Fig. 3 Color evolutionary tracks for the template galaxy spectral types. The tracks assume no luminosity evolution with redshift. Each point on the tracks represents a stepwise increase in $z$ of 0.05 .
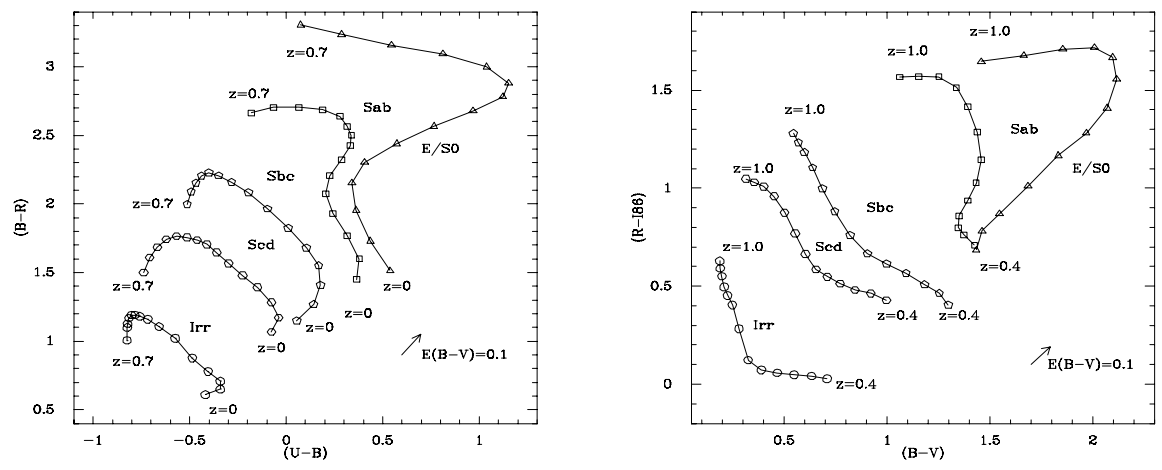
Fig. 4 Estimated photometric redshift vs. spectroscopically measured redshift for galaxies from high-redshift clusters ("+") and galaxies in the HOGPW survey ("X"). The diagonal represents the locus of perfect agreement between the two different measurements.

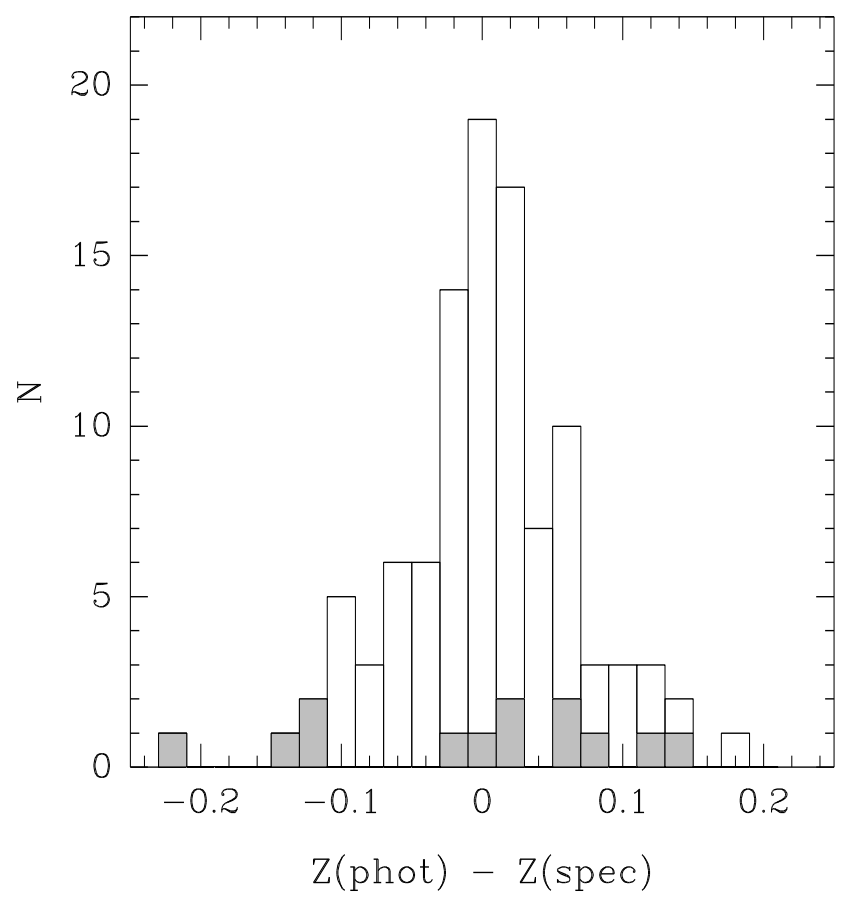


Fig. 5 Distribution of photometric redshift errors. Shaded bars: E+A galaxies in the sample, identified as described in the text.

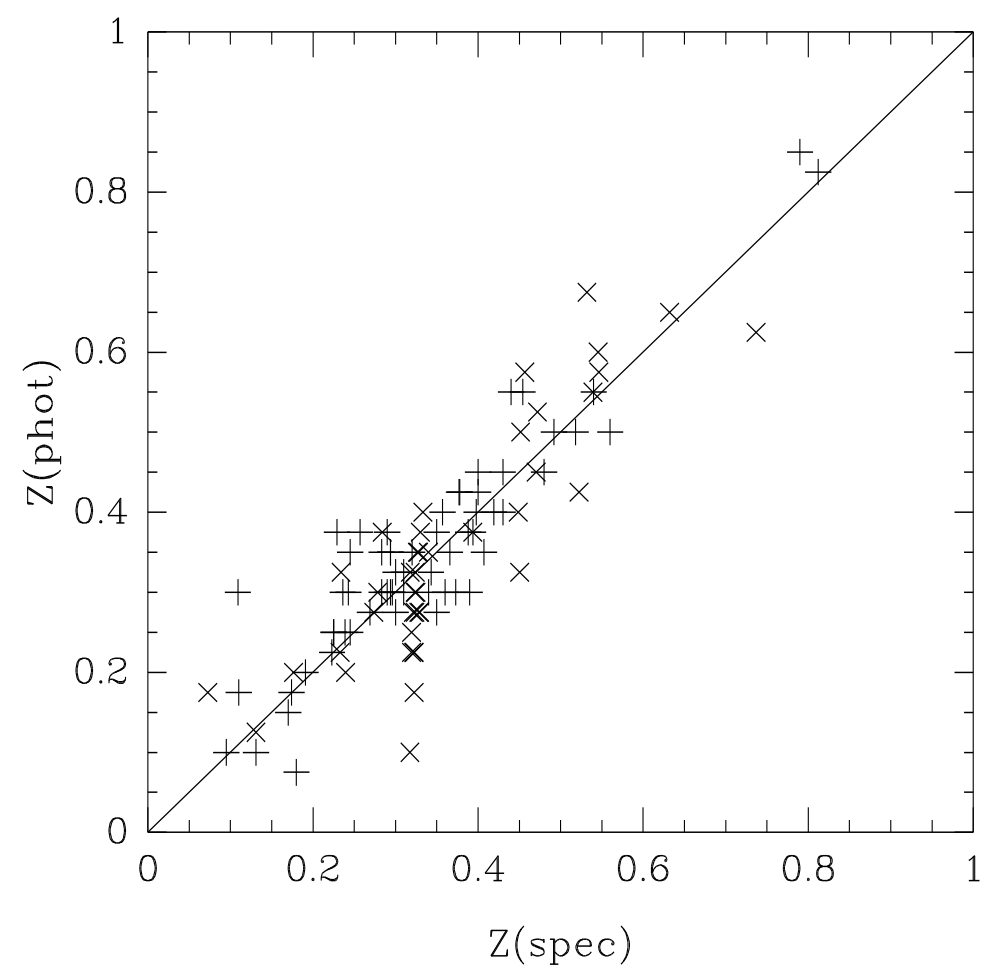


Fig. 6 Same as Fig. 3, but with color-color slices without U (left) or U and B (right) data. Although degeneracies at low redshift are serious, high redshift galaxy types are still well separated.
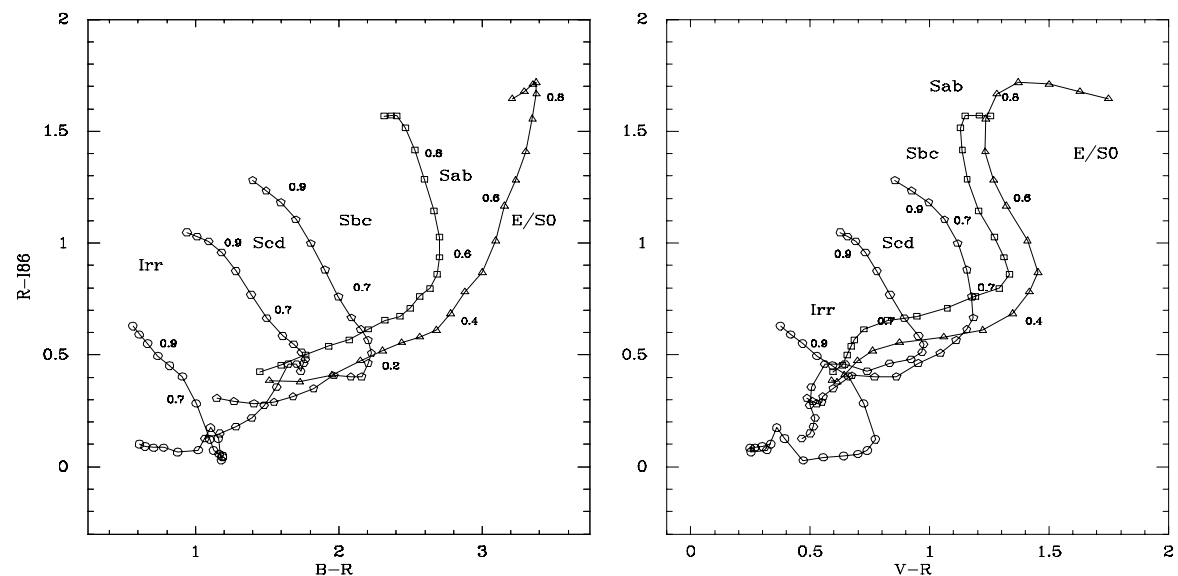